Article

\title{
Corynebacterium accolens Has Antimicrobial Activity against Staphylococcus aureus and Methicillin-Resistant S. aureus Pathogens Isolated from the Sinonasal Niche of Chronic Rhinosinusitis Patients
}

\author{
Martha Alemayehu Menberu ${ }^{1,2}$ (D), Sha Liu ${ }^{1}$, Clare Cooksley ${ }^{1}$, Andrew James Hayes ${ }^{3}$, Alkis James Psaltis ${ }^{1}$, \\ Peter-John Wormald ${ }^{1}$ and Sarah Vreugde ${ }^{1, *}$
}

1 Department of Surgery-Otolaryngology, Head and Neck Surgery, The University of Adelaide, Basil Hetzel Institute for Translational Health Research, Central Adelaide Local Health Network, Woodville 5011, Australia; martha.menberu@adelaide.edu.au (M.A.M.); sha.liu@adelaide.edu.au (S.L.); clare.cooksley@adelaide.edu.au (C.C.); alkis.psaltis@adelaide.edu.au (A.J.P.); peterj.wormald@adelaide.edu.au (P.-J.W.)

2 Department of Medical Microbiology, School of Biomedical and Laboratory Sciences, College of Medicine and Health Sciences, University of Gondar, Gondar 196, Ethiopia

3 Department of Microbiology and Immunology, Peter Doherty Institute for Infection and Immunity, University of Melbourne and The Royal Melbourne Hospital, Melbourne 3000, Australia; andrewhayes.bio@gmail.com

check for updates

Citation: Menberu, M.A.; Liu, S.; Cooksley, C.; Hayes, A.J.; Psaltis, A.J.; Wormald, P.-J.; Vreugde, S. Corynebacterium accolens Has Antimicrobial Activity against Staphylococcus aureus and Methicillin-Resistant S. aureus Pathogens Isolated from the Sinonasal Niche of Chronic Rhinosinusitis Patients. Pathogens 2021, 10, 207. https://doi.org/ $10.3390 /$ pathogens 10020207

Academic Editor: Bryan Heit

Received: 14 January 2021

Accepted: 11 February 2021

Published: 14 February 2021

Publisher's Note: MDPI stays neutral with regard to jurisdictional claims in published maps and institutional affiliations.

Copyright: (c) 2021 by the authors. Licensee MDPI, Basel, Switzerland. This article is an open access article distributed under the terms and conditions of the Creative Commons Attribution (CC BY) license (https:// creativecommons.org/licenses/by/ $4.0 /)$.
* Correspondence: sarah.vreugde@adelaide.edu.au; Tel.: +61-(0)8-8222-6928

\begin{abstract}
Corynebacterium accolens is the predominant species of the healthy human nasal microbiota, and its relative abundance is decreased in the context of chronic rhinosinusitis (CRS). This study aimed to evaluate the antimicrobial potential of $C$. accolens isolated from a healthy human nasal cavity against planktonic and biofilm growth of Staphylococcus aureus (S. aureus) and methicillin-resistant S. aureus (MRSA) clinical isolates (CIs) from CRS patients. Nasal swabs from twenty non-CRS control subjects were screened for the presence of $C$. accolens using microbiological and molecular techniques. C. accolens CIs and their culture supernatants were tested for their antimicrobial activity against eight S. aureus and eight MRSA 4CIs and S. aureus ATCC25923. The anti-biofilm potential of C. accolens cell-free culture supernatants (CFCSs) on S. aureus biofilms was also assessed. Of the 20 nasal swabs, $10 \mathrm{C}$. accolens CIs were identified and confirmed with rpoB gene sequencing. All isolates showed variable antimicrobial activity against eight out of 8 S. aureus and seven out of eight MRSA CIs. Culture supernatants from all C. accolens CIs exhibited a significant dose-dependent antibacterial activity $(p<0.05)$ against five out of five representative $S$. aureus and MRSA CIs. This inhibition was abolished after proteinase $\mathrm{K}$ treatment. $C$. accolens supernatants induced a significant reduction in metabolic activity and biofilm biomass of $S$. aureus and MRSA CIs compared to untreated growth control $(p<0.05)$. C. accolens exhibited antimicrobial activity against $S$. aureus and MRSA CIs in both planktonic and biofilm forms and holds promise for the development of innovative probiotic therapies to promote sinus health.
\end{abstract}

Keywords: chronic rhinosinusitis; Corynebacterium accolens; microbiota; sinus health

\section{Introduction}

Disruption of the human nasal microbiome homeostasis is found in patients with chronic rhinosinusitis (CRS). CRS is an inflammatory disorder of the mucosa of the nasal cavity and paranasal sinuses, characterized by various clinical manifestations including sinus/facial pain, nasal congestion, rhinorrhoea, post-nasal discharge, and a reduced sense of smell for a minimum of 12 weeks duration [1]. Whilst the aetiology of CRS is thought to be multifactorial, disruption of the microbial community residing in the sinuses, termed dysbiosis, has recently been implicated in CRS pathophysiology, in particular in more severe patients [2]. Dysbiosis is generally described as an imbalance of pathologic and commensal 
bacteria, which are involved in the protection against overgrowth of pathobionts or potentially disease-causing organisms [3,4]. In CRS patients, the microbiome is characterised by a decrease in the relative abundance of Corynebacterium and an expansion of pathogenic bacteria including Staphylococcus, Haemophilus, Moraxella and Enterobacteriacea [5]. Among pathogenic species, Staphylococcus aureus is the most frequently isolated species in patients with CRS [6]. Furthermore, exacerbations of CRS due to S. aureus and methicillin-resistant S. aureus (MRSA) have been reported in severe recalcitrant disease, leading to immune dysregulation, barrier dysfunction, biofilm formation, and worse clinical outcomes [7-9].

Several studies have shown that microbes that typically exist within the mucosa of the nasal cavity compete amongst themselves and inhibit the growth of competitors either by releasing antagonistic substances or by limiting access to nutrients from the surrounding environment [10,11]. For example, Corynebacterium accolens, a common commensal nasal species secretes the enzyme triacylglycerol lipase (LipS1) that degrades triacylglycerol to produce free fatty acids that interfere with the growth of Streptococcus pneumoniae in the nasal cavity [12,13].

Similarly, probiotic bacteria have been acknowledged as a potential novel treatment in various diseases of the gut linked to dysbiosis, as they can interfere with the growth of pathogenic organisms and provide a host-beneficial advantage [14]. In the context of CRS, manipulation of the sinonasal microbiome has been recognized as an innovative strategy to promote the re-establishment of sinonasal microbiome homeostasis and improve sinus health. As such, the potential of probiotic treatment in CRS has been demonstrated in in vivo models with several candidate bacterial species such as S. epidermidis and Lactobacillus sakei $[15,16]$. Similarly, intranasal administration of Corynebacterium species to carriers of $S$. aureus resulted in the eradication of $S$. aureus in $>70 \%$ of carriers [17].

To design a probiotic therapy to combat dysbiosis and help shape the microbiome in the context of CRS, a well-tolerated, safe and effective cocktail of beneficial microbes with good antimicrobial activity against pathobionts is required. A recent international sinonasal microbiome study compared the sinonasal microbiome of 410 controls and CRS patients, in which Corynebacterium was the most prevalent genus present in $>75 \%$ of CRS patients and controls with a significant reduction in its relative abundance in CRS patients compared to controls [5]. Together with the notion that Corynebacterium species can interfere with the growth of pathogens $[12,13,17]$, these findings support the probiotic potential of Corynebacterium species. However, Corynebacterium species have also been reported to mediate sinusitis [16]. Therefore, it is important to define the commensal status of Corynebacteria at species and strain-level along with their interaction against the most prevalent CRS pathogens, particularly S. aureus and MRSA.

This study was designed to isolate and characterize Corynebacterium accolens strains from healthy sinonasal cavities and evaluate their antimicrobial and antibiofilm potential against S. aureus and MRSA clinical isolates from CRS patients.

\section{Results}

A total of 36 study subjects, 20 non-CRS controls ( 8 males and 12 females, aged between 20-70 years old) and 16 CRS patients ( 8 males and 8 females, aged between 36-90 years old) were included to collect nasal swab samples and identify the clinical isolates. The demographic characteristics and clinical data of each study subject are summarized in Table 1. 
Table 1. Demographic and clinical characteristics of the study subjects.

\begin{tabular}{ccc}
\hline Characteristic & Non-CRS Controls, No. (\%) & Patients with CRS, No. (\%) \\
\hline Number of subjects & 20 & 16 \\
Mean age (years) & 45.7 & 64.0 \\
Gender (M/F) & $8 / 12$ & $8 / 8$ \\
Active smoker & $0(0)$ & $1(6.3)$ \\
Asthma & $6(30)$ & $8(50)$ \\
Diabetes mellitus & $1(5)$ & $0(0)$ \\
Cystic fibrosis & $0(0)$ & $0(0)$ \\
GERD & $6(30)$ & $3(18.8)$ \\
Aspirin sensitivity & $0(0)$ & $3(18.8)$ \\
Tonsillitis in the past 6 months & $0(0)$ & $0(0)$ \\
Ear infection in the past 6months & $0(0)$ & $0(0)$ \\
Nasal polyposis & $0(0)$ & $7(43.8)$ \\
\hline Abbreviations: CRS, chronic rhinosinusitis; F, female;
\end{tabular}

Abbreviations: CRS, chronic rhinosinusitis; F, female; GERD, gastroesophageal reflux; $\mathrm{M}$, male.

Based on the phenotypic API 20 Staph and chromogenic MRSA selective agar screening methods, 16 S. aureus isolates (8 methicillin-sensitive S. aureus (MSSA) and 8 MRSA) were identified from 16 CRS patients. Characteristics of $S$. aureus clinical isolates used in this study are shown in Table S2.

\subsection{Identification of $C$. accolens Isolates}

From 20 non-CRS controls, $10 \mathrm{C}$. accolens isolates were identified by the API Coryne test kit with $\geq 90.0 \%$ similarity to known strains of $C$. accolens from the database. The molecular identification of all C. accolens isolates with the PCR amplification followed by gel electrophoresis resulted in a DNA fragment of approximately $446 \mathrm{bp}$ in size (Figure S1). In order to confirm the strain level identification, partial rpoB gene sequencing was done for all C. accolens isolates. As shown in Table 2, the $r p o B$ nucleotide sequence BLAST of 10 C. accolens isolates showed $96 \%$ to $100 \%$ similarity and $99 \%$ to $100 \%$ query coverage with the known culture collection strain, C. accolens CIP 104783 (from the Pasteur Institute Collection, Biological Resource Center of Pasteur Institute (CRBIP), Paris, France), GenBank accession number AY492242 identified previously [18].

Table 2. Identification of Corynebacterium accolens using API Coryne 20 test system and rpoB gene sequencing.

\begin{tabular}{|c|c|c|c|c|c|}
\hline \multirow{2}{*}{$\begin{array}{l}\text { Isolate } \\
\text { Code }\end{array}$} & \multirow{2}{*}{$\begin{array}{l}\text { API Coryne } 20 \text { Identification } \\
(\% \text { Similarity })\end{array}$} & \multicolumn{4}{|c|}{ rpoB Gene Sequence Identification } \\
\hline & & Strains & $\%$ Similarity & $\%$ Query Coverage & Accession Number \\
\hline $\mathrm{C} 778$ & C. accolens $(90.0)$ & C. accolens & 98.3 & 100 & MT856944 \\
\hline C779 & C. accolens (95.6) & C. accolens & 96.0 & 100 & MT856945 \\
\hline $\mathrm{C} 780$ & C. accolens (90.0) & C. accolens & 97.6 & 100 & MT856946 \\
\hline C781 & C. accolens (99.4) & C. accolens & 98.7 & 100 & MT856947 \\
\hline C782 & C. accolens (95.6) & C. accolens & 99.5 & 100 & MT856948 \\
\hline C783 & C. accolens (90.0) & C. accolens & 98.2 & 99 & MT856949 \\
\hline C784 & C. accolens (91.4) & C. accolens & 98.3 & 100 & MT856950 \\
\hline C785 & C. accolens (90.0) & C. accolens & 96.6 & 100 & MT856951 \\
\hline C786 & C. accolens (90.0) & C. accolens & 97.3 & 100 & MT856952 \\
\hline C787 & C. accolens (90.0) & C. accolens & 96.4 & 100 & MT856953 \\
\hline
\end{tabular}

Note: ${ }^{+}$Results were interpreted based on various biochemical reactions on the API Coryne test strip and \% similarity of the isolates were identified by comparing with $C$. accolens isolates deposited from the database (V4.0) using the apiweb ${ }^{\mathrm{TM}}$ software.

\subsection{Phylogenetic Relationship of the Strains}

Comparison of the $r p o B$ gene sequences with the corresponding $C$. accolens sequences from the GenBank database showed that $C$. accolens strains were placed in the evolutionary clade of Corynebacterium origin. Strains of C. accolens, C778 and C779 were clustered together with strains C784 and C785, respectively, with a bootstrap value of $100 \%$. Furthermore, C. accolens strain C782 was clustered with a culture collection strain, C. accolens ATCC 49726 
with a bootstrap value of $99 \%$. The phylogenetic analysis based on the rpoB genes of all C. accolens clinical isolates and their closest related Corynebacteria species are indicated in Figure 1.

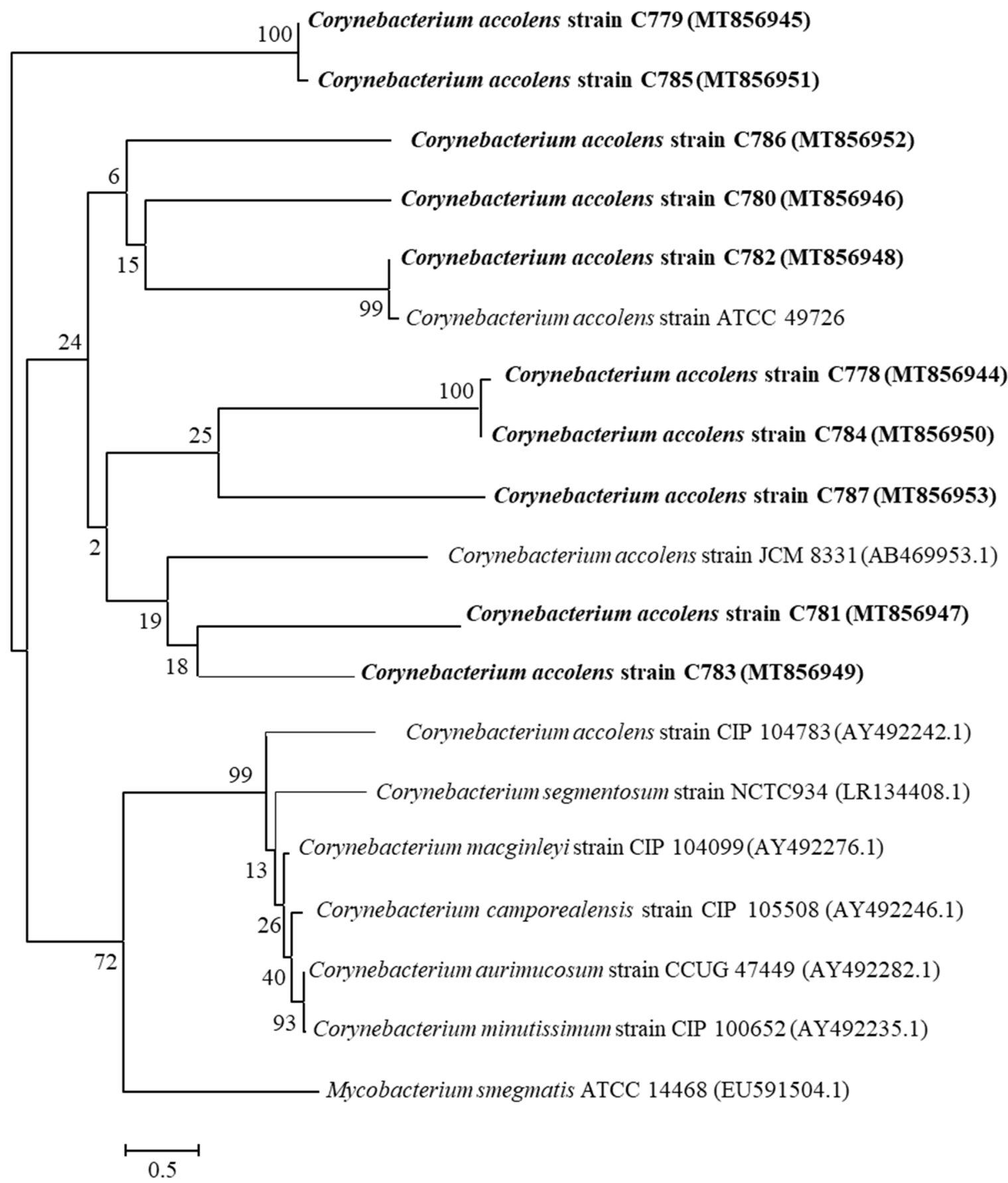

Figure 1. Phylogenetic tree showing the evolutionary relationships between $10 \mathrm{C}$. accolens nasal isolates, a reference strain C. accolens ATCC49726 and the type strains of related species (C. accolens CIP 104783, C. accolens JCM 8331 and other Corynebacteria species) based on rpoB gene sequences analysed using the neighbour-joining method. The percentages of replicate trees in which the associated taxa clustered together in the bootstrap test (1000 replicates) are shown next to the branches. Numbers in parentheses represent the sequence accession number in GenBank. Mycobacterium smegmatis ATCC 14468 was used as an outgroup. The scale bar represents 0.5 -nucleotide substitutes per position. 


\subsection{Spectrum of Antimicrobial Activity}

Eight methicillin-sensitive $S$. aureus (MSSA) and eight methicillin-resistant $S$. aureus (MRSA) were retrieved from the nasal cavity of CRS patients.

In the deferred growth inhibition assay all, of the $C$. accolens strains showed antagonistic effects against most MSSA and MRSA clinical isolates, but the degree of antagonism varied among the $C$. accolens strains. From all S. aureus clinical isolates, only one MRSA strain could not be inhibited by any of the C. accolens strains. Most of the $C$. accolens strains showed low inhibitory activities against various strains of MSSA, MRSA and reference strain S. aureus ATCC 25923 (inhibition zones of less than $5 \mathrm{~mm}$ ). Interestingly, three of the isolated strains (C. accolens C779, C. accolens C781 and C. accolens C787), exhibited strong inhibition on the growth of MSSA C26 and MRSA C261 (inhibition zones of more than $8 \mathrm{~mm}$ ) (Figure 2). C. accolens C781 was the most effective strain in inhibiting the growth of eight of eight (100\%) MSSA and six of eight (75.0\%) MRSA CIs tested. In contrast, C. accolens C782 was the least effective strain, showing inhibitory activities against only four of eight (50.0\%) MSSA and two of eight (25.0\%) MRSA CIs tested. Results are summarized in Table 3.

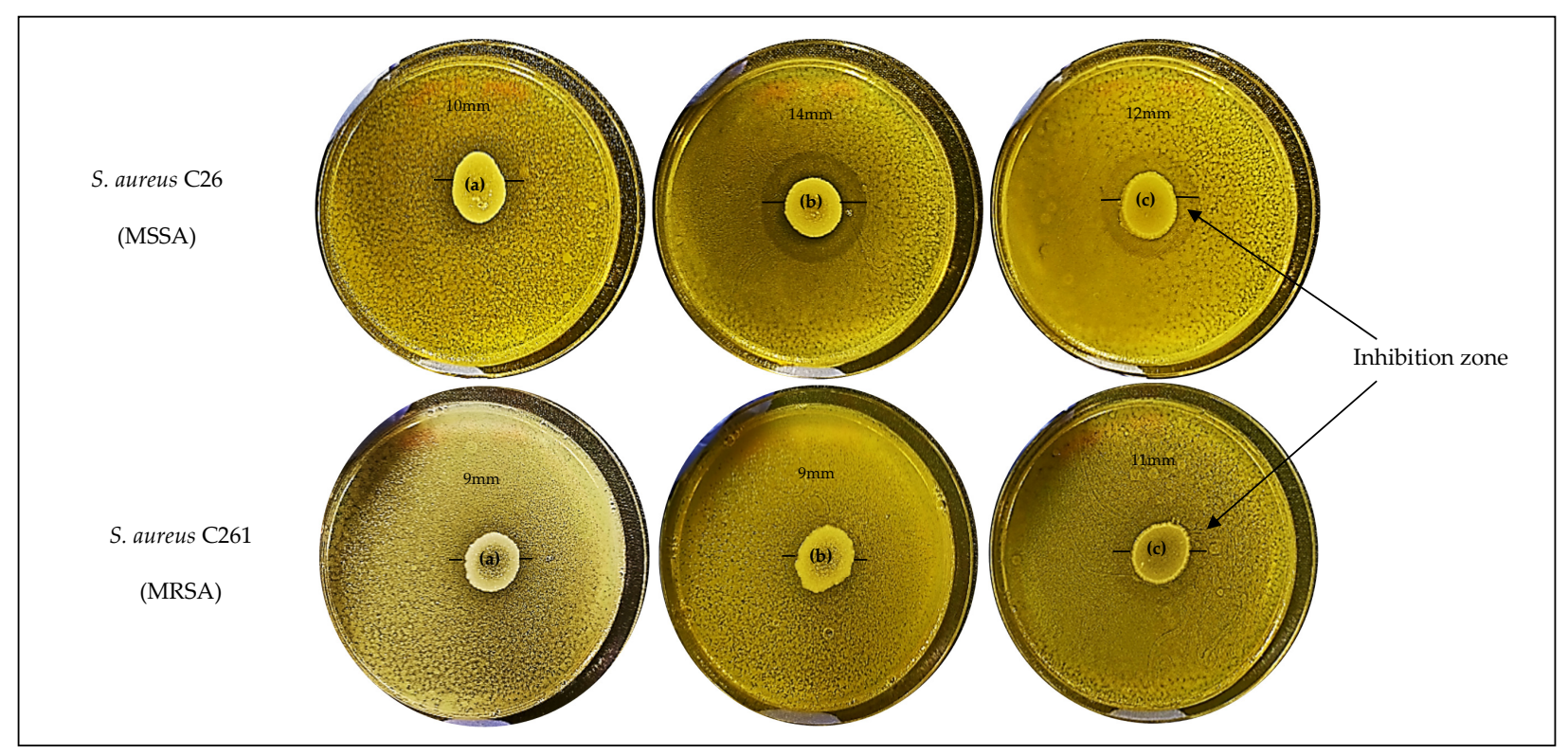

Figure 2. Antagonistic activity of selected C. accolens nasal isolates (a) C. accolens C779, (b) C. accolens C781 and (c) C. accolens C787 spotted on a lawn of S. aureus clinical isolates, MSSA C26 (top image) and MRSA C261 (bottom image) on tryptone soya agar (TSA) medium. The inhibition zone diameter was measured in at least three replicate experiments and the mean values were taken to score the extent of inhibition. The single line represents the growth inhibition zone. CI, Clinical isolate; MSSA, methicillin-sensitive S. aureus; MRSA, methicillin-resistant S. aureus. 
Table 3. Antagonistic activity of $C$. accolens against S. aureus clinical isolates in deferred growth inhibition assay.

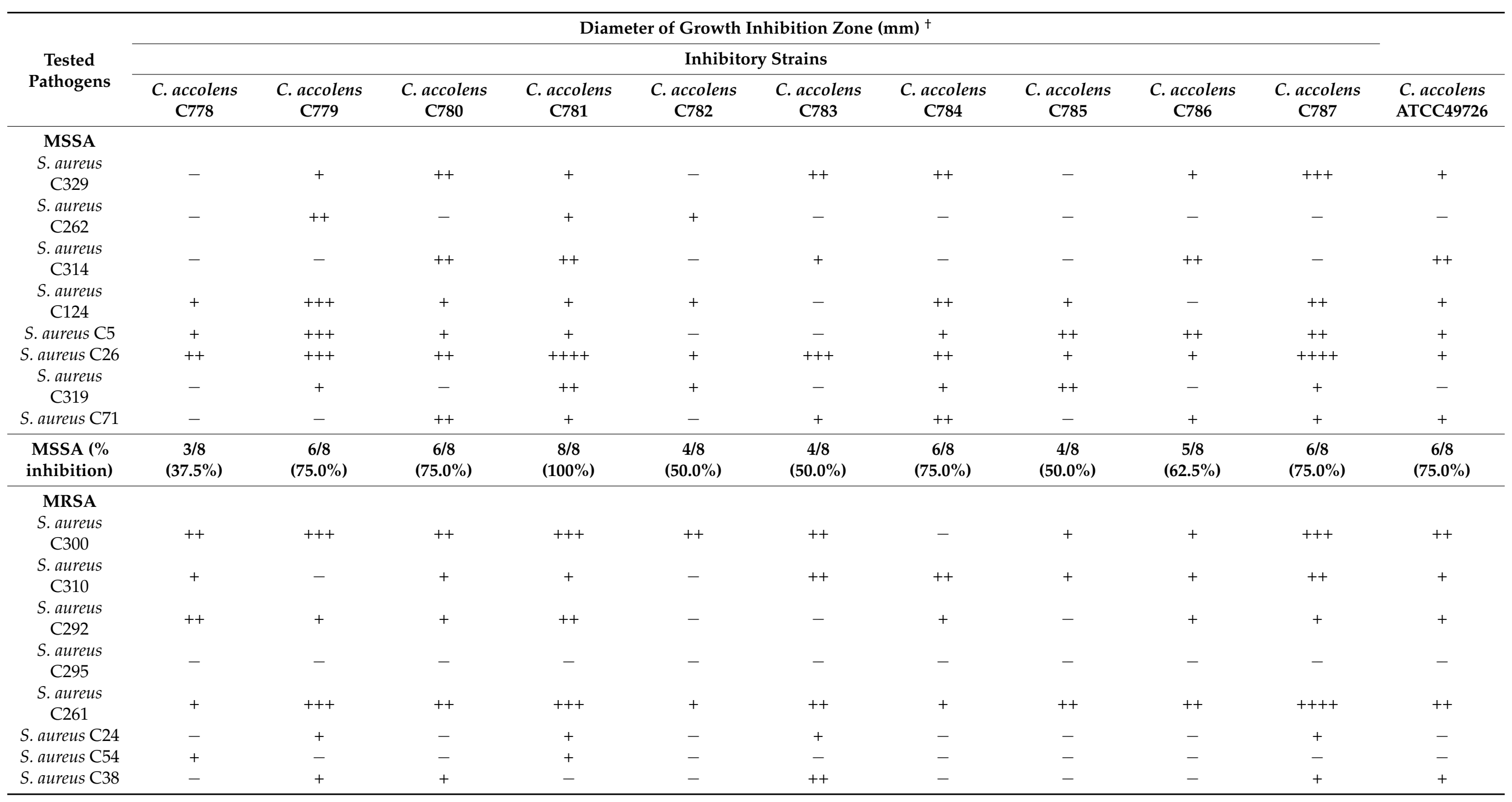


Table 3. Cont.

\begin{tabular}{|c|c|c|c|c|c|c|c|c|c|c|c|}
\hline \multirow{3}{*}{$\begin{array}{c}\text { Tested } \\
\text { Pathogens }\end{array}$} & \multicolumn{10}{|c|}{ Diameter of Growth Inhibition Zone $(\mathrm{mm})^{+}$} & \multirow[b]{3}{*}{$\begin{array}{l}\text { C. accolens } \\
\text { ATCC49726 }\end{array}$} \\
\hline & \multicolumn{10}{|c|}{ Inhibitory Strains } & \\
\hline & $\begin{array}{l}\text { C. accolens } \\
\text { C778 }\end{array}$ & $\begin{array}{l}\text { C. accolens } \\
\text { C779 }\end{array}$ & $\begin{array}{l}\text { C. accolens } \\
\text { C780 }\end{array}$ & $\begin{array}{l}\text { C. accolens } \\
\text { C781 }\end{array}$ & $\begin{array}{l}\text { C. accolens } \\
\text { C782 }\end{array}$ & $\begin{array}{l}\text { C. accolens } \\
\text { C783 }\end{array}$ & $\begin{array}{l}\text { C. accolens } \\
\text { C784 }\end{array}$ & $\begin{array}{l}\text { C. accolens } \\
\text { C785 }\end{array}$ & $\begin{array}{l}\text { C. accolens } \\
\text { C786 }\end{array}$ & $\begin{array}{c}\text { C. accolens } \\
\text { C787 }\end{array}$ & \\
\hline $\begin{array}{c}\text { MRSA (\% } \\
\text { inhibition) }\end{array}$ & $\begin{array}{c}5 / 8 \\
(62.5 \%)\end{array}$ & $\begin{array}{c}5 / 8 \\
(62.5 \%)\end{array}$ & $\begin{array}{c}5 / 8 \\
(62.5 \%)\end{array}$ & $\begin{array}{c}6 / 8 \\
(75.0 \%)\end{array}$ & $\begin{array}{c}2 / 8 \\
(25.0 \%)\end{array}$ & $\begin{array}{c}5 / 8 \\
(62.5 \%)\end{array}$ & $\begin{array}{c}3 / 8 \\
(37.5 \%)\end{array}$ & $\begin{array}{c}3 / 8 \\
(37.5 \%)\end{array}$ & $\begin{array}{c}4 / 8 \\
(50.0 \%)\end{array}$ & $\begin{array}{c}6 / 8 \\
(75.0 \%)\end{array}$ & $\begin{array}{c}5 / 8 \\
(75.0 \%)\end{array}$ \\
\hline $\begin{array}{c}\text { S. aureus } \\
\text { ATCC25923 }\end{array}$ & + & + & - & + & + & - & + & + & + & + & + \\
\hline $\begin{array}{c}\text { Total } \\
(\% \\
\text { inhibition) }\end{array}$ & $\begin{array}{c}9 / 17 \\
(52.9 \%)\end{array}$ & $\begin{array}{c}12 / 17 \\
(70.6 \%)\end{array}$ & $\begin{array}{c}11 / 17 \\
(64.7 \%)\end{array}$ & $\begin{array}{c}15 / 17 \\
(88.2 \%)\end{array}$ & $\begin{array}{c}7 / 17 \\
(41.2 \%)\end{array}$ & $\begin{array}{c}9 / 17 \\
(52.9 \%)\end{array}$ & $\begin{array}{c}10 / 17 \\
(58.8 \%)\end{array}$ & $\begin{array}{c}8 / 17 \\
(47.1 \%)\end{array}$ & $\begin{array}{c}10 / 17 \\
(58.8 \%)\end{array}$ & $\begin{array}{c}13 / 17 \\
(76.5 \%)\end{array}$ & $\begin{array}{c}12 / 17 \\
(70.5 \%)\end{array}$ \\
\hline
\end{tabular}

Note: ${ }^{\dagger}$ The extent of inhibition was scored based on the inhibition zone diameter result as: $-(0 \mathrm{~mm}),+(<5 \mathrm{~mm}),++(5-7 \mathrm{~mm}),+++(8-10 \mathrm{~mm})$ and $++++(>10 \mathrm{~mm})$. 


\subsection{Inhibitory Activity of C. accolens Concentrated Cell-Free Culture Supernatants (CFCSs)}

All of the C. accolens cell-free culture supernatants in the present study exhibited a significant dose-dependent antibacterial activity against all of the $S$. aureus isolates tested (MSSA C5, MSSA C26, MRSA C300, MRSA C261 and ATCC 25923) compared to the control group consisting of $S$. aureus in TSB without CFCS $(p<0.05)$. The highest concentration of $90 \%$ CFCS in TSB from all C. accolens strains significantly inhibited the planktonic growth of all $S$. aureus isolates $(p<0.0001)$ compared to untreated controls. However, lower concentrations of $30 \%$ CFCS in TSB $(102 \mu \mathrm{g} / \mathrm{mL})$ did not show antibacterial activity against any of the $S$. aureus isolates tested $(p>0.05)$ (Figure 3$)$.
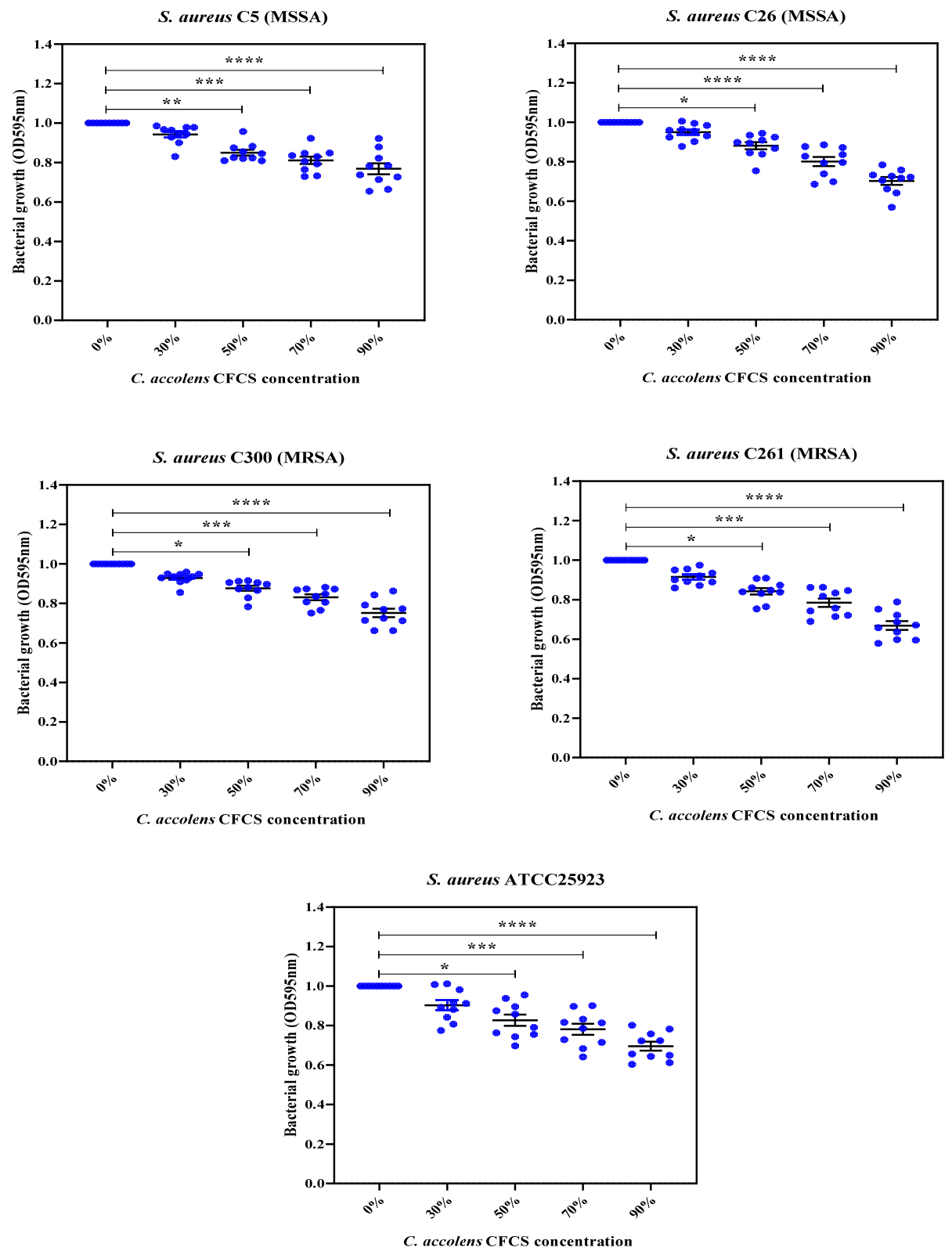

Figure 3. Growth inhibitory effect of $C$. accolens CFCSs $(n=10$, represented by blue dots) against S. aureus (2 MSSA (C5, C26), 2 MRSA (C300, C261) and S. aureus ATCC25923) at various concentrations, $30 \%, 50 \%, 70 \%$ and $90 \%$ (CFCSs diluted in TSB), compared to controls (corresponding volume of $\mathrm{TSB}+\mathrm{SA}$, normalized to $1.0 \mathrm{OD}$ value) following $24 \mathrm{~h}$ treatment. The results are expressed as means \pm SEM of three independent experiments. SA, S. aureus; CI, clinical isolate; One-way ANOVA ${ }^{*} p<0.05 ;{ }^{* *} p<0.01 ;{ }^{* * *} p<0.001 ;{ }^{* * *} p<0.0001$; SEM, standard error of the means. 
C. accolens $\mathrm{C779}, \mathrm{C781}$ and C787 showed the highest antagonistic activity in the deferred growth inhibition assay, and their CFCSs demonstrated a strong anti-staphylococcal activity for the three S. aureus test strains (MSSA C26, MRSA C261 and ATCC 25923) and were selected for investigation of the dose-dependent growth inhibition of their CFCSs. The highest antimicrobial activity was observed for C. accolens C781 CFCS at all tested concentrations ( $30 \%$ to $90 \%$ ) compared to controls $(p<0.05)$. C. accolens C779 and C. accolens C787 CFCSs showed significant growth inhibition at concentration of CFCS in TSB higher than 50\% against MRSA C261 $(p<0.05)$ and 70\% CFCS in TSB against MSSA C26 and S. aureus ATCC 25923 strains (Figure $4 \mathrm{~A}-\mathrm{C}$ ).
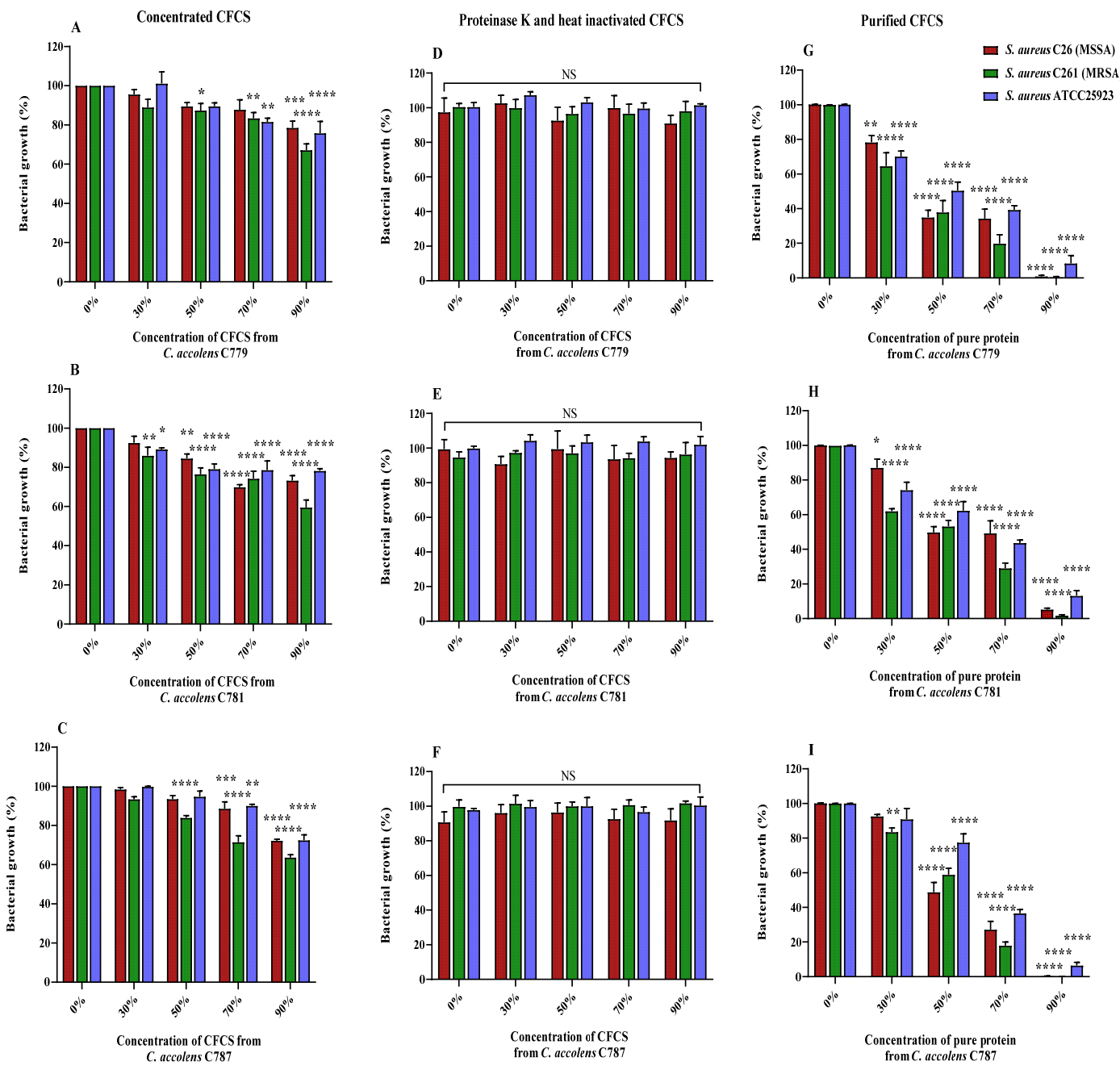

Figure 4. Antibacterial potential of C. accolens purified CFCSs (extracted from strains C779, C781 and C787) against S. aureus planktonic cells [MSSA C26 (red), MRSA C261 (green) and ATCC25923 (blue)]. (A-C) represents treatment of S. aureus strains with concentrated CFCSs from C. accolens strains C779 (A), C781 (B) and C787 (C) at concentrations of 30-90\% or a positive control (corresponding bacterial inoculum in TSB); (D-F) represents treatment of $S$. aureus strains with proteinase $\mathrm{K}$ and heat inactivated CFCSs from C. accolens strains C779 (D), C781 (E) and C787 (F) at concentrations of 30-90\% or a positive control (corresponding bacterial inoculum in concentrated TSB treated with proteinase K and heat); (G-I) represents treatment of S. aureus strains with purified CFCSs from C. accolens strains C779 (G), C781 (H) and C787 (I) at concentrations of $30-90 \%$ or a positive control (corresponding bacterial inoculum in TSB and MilliQ water, normalized to the water-diluted TSB). Bacterial growth (\%) was determined as follows: [(Abs growth control-Abs CFCS treated)/Abs growth control] $\times 100$; where, $\mathrm{Abs}=$ mean absorbance. Data presented as means \pm SEM of three independent experiments. PK, proteinase K; CTSB, concentrated tryptone soya broth; SA, S. aureus; CI, clinical isolate; One-way ANOVA ${ }^{*} p<0.05 ;{ }^{* *} p<0.01 ;{ }^{* * *} p<0.001$; **** $p<0.0001$; NS, not significant; SEM, standard error of the mean. 


\subsection{Characterization of the Inhibitory Effect of CFCSs Produced by C. accolens Strains}

\subsubsection{Effect of Proteinase K and Heat Inactivation}

The nature of the inhibitory substance produced by selected C. accolens strains was studied by treating their CFCSs with proteinase $\mathrm{K}$ and heat. CFCSs from the 3 selected C. accolens strains, C779, C781 and C787, at different concentrations completely lost their antimicrobial activity against the selected $S$. aureus strains after treatment with proteinase $\mathrm{K}(1 \mathrm{mg} / \mathrm{mL})$ followed by heat $\left(55^{\circ} \mathrm{C}, 30 \mathrm{~min}\right)$ (Figure $\left.4 \mathrm{D}-\mathrm{F}\right)$. This indicated that inhibitory effects of the $C$. accolens strains were due to the proteinaceous nature of active substances.

\subsubsection{Effect of Purified Protein Treatment}

Purified protein extracts from the selected C. accolens CFCSs showed concentrationdependent inhibitory activity against the tested $S$. aureus strains as indicated in Figure 4G-I $(p<0.05)$. Purified protein extracts from C. accolens strains C779 and C781 at 30\% inhibited the growth of both MSSA C26 $(p<0.05)$, MRSA C261 and ATCC 25923 compared to control $(p<0.0001)$. Higher or $90 \%$ concentration of purified protein extracts from all tested C. accolens strains, C779, C781 and C787, exhibited a stronger antimicrobial effect $(p<0.0001)$ against all S. aureus strains compared to control.

\subsection{C. accolens CFCS Inhibits S. aureus and MRSA Biofilm Metabolic Activity}

The activity of $C$. accolens CFCS on the metabolic activity of 48-h biofilms formed by 3 representative S. aureus strains (MSSA C26, MRSA C261 and ATCC 25923) was evaluated using alamarBlue assays. As shown in Figure 5A-C, CFCSs obtained from C. accolens C779, C781 and C787 had a concentration-dependent reduction in metabolic activity of both MSSA C26 and MRSA C261 CIs in established biofilms with values reduced by $23 \%$ to $42 \%$ compared to respective positive control. Biofilm of S. aureus ATCC25923 could be inhibited by about $26 \%$ to $29 \%$ compared to control by C. accolens C781and C787 CFCS at concentrations ranging between $70 \%$ and $90 \%$. However, only high concentrations of CFCS of $90 \%$ exhibited a significant inhibitory effect on biofilms formed by MSSA C26, MRSA C261 and S. aureus ATCC25923. The C. accolens CFCS exhibited different anti-biofilm activity against the 3 S. aureus strains tested.

\subsection{C. accolens CFCS Reduces S. aureus and MRSA Biofilm Biomass}

Figure 5D-F show the effects of CFCSs extracted from C. accolens strains, C779, C781 and C787 on the S. aureus biofilm biomass established by clinical isolates MSSA C26 and MRSA C261 and reference strain ATCC 25923. Although the biofilm of MRSA C261 was less affected than MSSA C26, all tested C. accolens CFCSs at the highest concentration (90\%) reduced the biofilm biomass of both S. aureus clinical isolates (between $28 \%$ and $40 \%$ ). However, the S. aureus ATCC 25923 biofilm biomass was not affected by CFCS at any of the concentrations tested. 

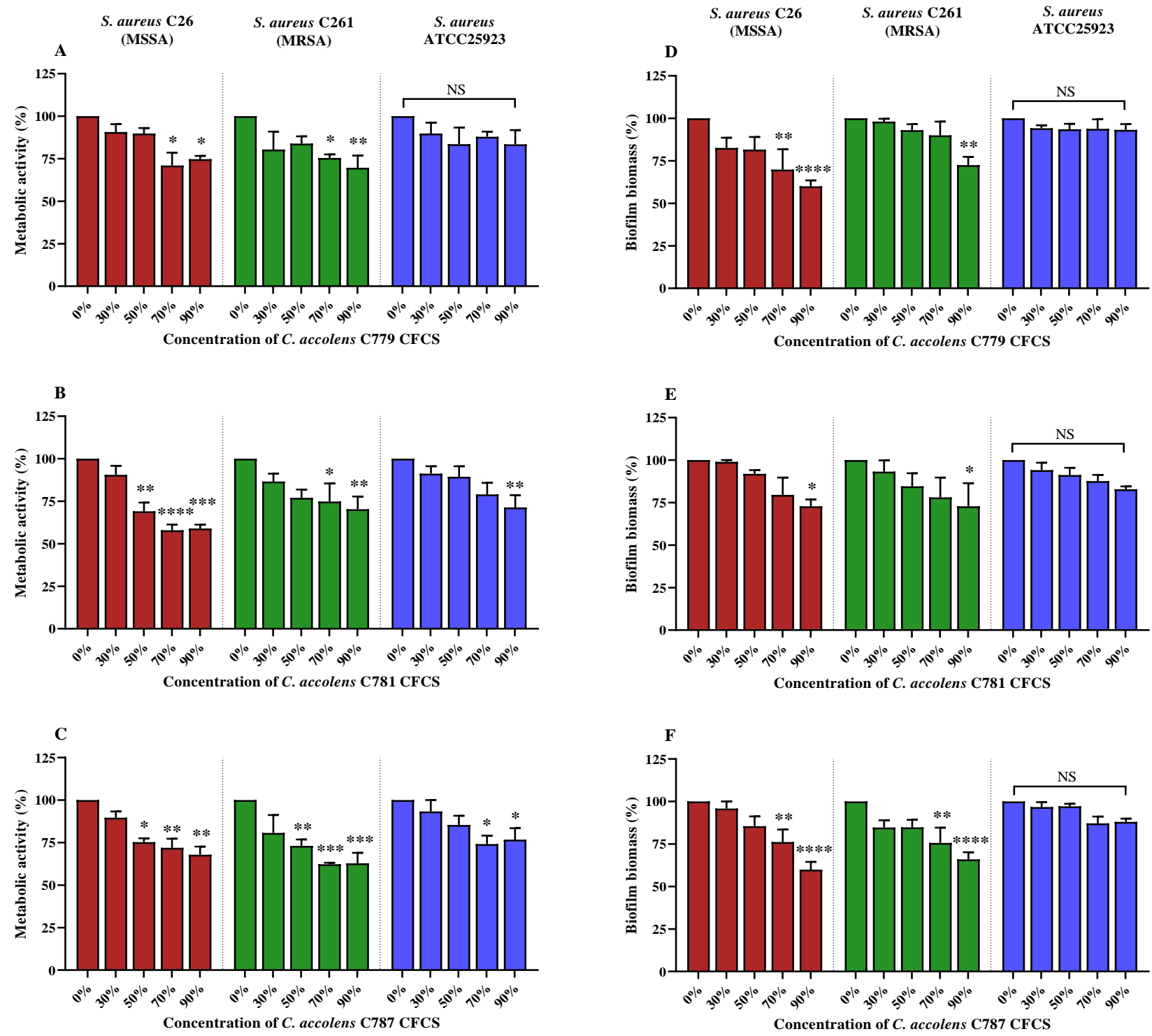

Figure 5. Anti-biofilm potential of C. accolens CFCS (extracted from strains C779, C781 and C787) on S. aureus biofilms established by clinical isolates (MSSA C26 (red), MRSA C261 (green) and ATCC25923 (blue)). (A-C) represent reduction of metabolic activity of biofilms formed by S. aureus strains (C26, C261 and ATCC25923) normalised to positive control (TSB + corresponding bacterial inoculum) in the presence and absence of CFCS from C. accolens strains, C779 (A), C781 (B) and C787 (C) diluted with TSB at different concentrations (30\%, 50\%, 70\% and 90\%). (D-F) represent biofilm biomass reduction of S. aureus strains (C26, C261 and ATCC25923) normalised to positive control (TSB + corresponding bacterial inoculum) after $24 \mathrm{hrs}$ incubation with CFCS at different concentrations (30\%, 50\%, 70\% and 90\%) extracted from 3 C. accolens strains, C779 (D), C781 (E) and C787 (F). Values represent the means \pm SEM of at least three independent experiments. Metabolic activity $(\%$ inhibition $)=(($ FI growth control - FI CFCS treated $) /$ FI growth control $) \times 100$, where FI = average fluorescence intensity, and Biofilm biomass $(\%$ reduction $)=(($ Abs growth control-Abs CFCS treated $) /$ Abs growth control $) \times 100 ;$ where Abs = mean absorbance. SA, S. aureus; CI, clinical isolate; One-way ANOVA ${ }^{*} p<0.05 ;{ }^{* *} p<0.01 ;{ }^{* * *} p<0.001 ;{ }^{* * * *} p<0.0001$; NS, not significant; SEM, standard error of the mean.

\section{Discussion}

This study indicates the probiotic potential of C. accolens with the potential of this species to help shape a dysbiotic microbiome in the context of CRS by interfering with the growth of MSSA and MRSA in planktonic and biofilm form. Some beneficial nasal bacteria have been evidenced in providing beneficial functions to restore the sinonasal microbiome 
composition and improving immune health in patients with CRS through direct pathogen inhibition, secretion of a bioactive molecule or nutrient competition [19]. Our results show that $C$. accolens strains isolated from the sinonasal cavities of non-CRS control patients have antimicrobial activity against MSSA and MRSA strains isolated from the sinonasal cavities of CRS patients. Both MSSA and MRSA planktonic cells and biofilms were sensitive to $C$. accolens and our results indicate a secreted protein to likely be responsible for this activity. Although all $C$. accolens strains had anti-staphylococcal activity, there was a strain-dependent variability in the host range and strength of anti-microbial action.

The human nasal cavity forms a complex microbial ecosystem colonized by several resident microorganisms comprising both commensals and pathobionts [20]. Emerging evidence indicates that Corynebacteria are the predominant genus in the sinonasal niche present in $>75 \%$ of CRS patients and controls; however, the relative abundance of Corynebacteria is reduced in patients with CRS [5]. The reduction of Corynebacteria, and increased relative abundance of pathobionts such as $S$. aureus in these patients, reflect a potentially disturbed host-microbe-microbe balance that might contribute to the pathophysiology of this disease $[2,5,21]$. From those studies, it appears that Corynebacteria can be in general regarded as a commensal in the sinonasal cavities. This is also in line with our study where C. accolens was isolated from the sinonasal cavities of at least $50 \%$ of healthy controls. However, an outgrowth of Corynebacterium species has also been implicated in CRS [16]. Therefore, it is important to define the commensal status of Corynebacteria at the species level. Sequencing of $r p o B$ and $16 \mathrm{~S}$ rRNA genes are the most widely used molecular methods for reliable identification of Corynebacterium species, and the rpoB gene is considerably more polymorphic than the $16 \mathrm{~S}$ rRNA gene for members of the genus Corynebacterium [18,22]. In this study, rроB gene sequencing confirmed that all isolated Corynebacterium strains had a pairwise sequence similarity of $96 \%$ to $100 \%$ with a culture collection strain $C$. accolens CIP 104783, classifying them as C. accolens. Our phylogenetic analysis based on the rpoB gene sequences also revealed that some strains such as (C779 and C785), (C778 and C784) and (C781 and C783) were closely related and shared the same clade. Potentially due to their close phylogenetic relationship, these strains tended to have similar antimicrobial properties against $S$. aureus and MRSA.

In this study, all ten C. accolens strains were active against a variety of $S$. aureus and MRSA strains. Notably, three of the strains designed as C779, C781 and C787 showed strong inhibition against at least 6/8 (75\%) MSSA and 5/8 (62.5\%) MRSA CIs tested. In particular, $C$. accolens strain $C 781$ had the widest host range and exhibited inhibitory activity against eight out of eight (100\%) S. aureus and six out of eight (75\%) MRSA CIs. Given that the antimicrobial properties appear similar in MSSA and MRSA, we speculate that the molecular mechanism is likely unrelated to known mechanisms of antibiotic resistance. An increasing amount of research has shown an inverse correlation between Corynebacterium and S. aureus nasal colonization [23-25]. For example, in a cohort of forty healthy adults, C. accolens negatively correlated with $S$. aureus colonization and positively correlated with C. pseudodiphtheriticum [23]. Moreover, a previous study by Uehara Y et al. described that frequently implanting Corynebacterium species eradicated S. aureus colonization in 12 of 17 healthy adult carriers, suggesting the beneficial role of Corynebacterium in the abolition of $S$. aureus nasal colonization [17]. Despite the complexity of Corynebacterium-S. aureus interactions and strain-level variations, those studies are in line with the present study and support the possibility of commensal C. accolens strains to be used as probiotic therapy in the context of CRS.

Some studies have also focused on the activity of antibacterial products in commensal Corynebacterium CFCSs toward pathogens. For example, a secreted factor by C. pseudodiphtheriticum, a closely related Corynebacterium species to C. accolens, has revealed bactericidal activity against various $S$. aureus strains including MRSA [24]. In our study, the antimicrobial effects by $C$. accolens against $S$. aureus are at least in part due to a secreted antimicrobial substance. Moreover, given the abrogation of this effect by treatment of the CFCS with Proteinase $\mathrm{K}$ and heat, the bioactive product is likely a protein or peptide. The 
commensal-derived products in a complex sinonasal niche can directly act on challenging the pathogenic bacteria to maintain a well-balanced microbiome. Recently, a novel peptide antibiotic termed lugdunin produced by the nasal and skin commensal Staphylococcus lugdunensis has demonstrated strong bactericidal activity against $S$. aureus nasal and skin colonization as well as the immunomodulatory potential to protect the host [26].

The inhibitory activity of C. accolens, has been previously reported against S. pneumoniae and was mainly due to the production of primary triacylglycerol lipase and release of anti-pneumococcal free fatty acids from representative human nostril and skin surface triacylglycerols [13]. Furthermore, a previous study done by Ramsey MM et al. demonstrated another possibility of interaction between commensal Corynebacterium species and $S$. aureus pathobionts with a view to managing $S$. aureus nasal colonization. In this study, the virulence of $S$. aureus was heavily affected by commensal C. amycolatum, C. accolens, and C. pseudodiphtheriticum through altered expression of the $S$. aureus quorum sensing-controlled accessory gene regulator $(a g r)$ genetic locus involved in colonization and virulence, and shifting bacterial behavior from virulence to a commensal lifestyle [25]. It is particularly interesting to note that the inhibitory activity of commensal C. accolens strains in our study is more likely due to proteins affecting the growth of several MSSA and MRSA isolates that are pathogenic in CRS. However, more in-depth studies are needed to identify and characterize the $C$. accolens secreted protein that is responsible for the observed antimicrobial effect and to investigate the unexplored mechanism of action.

It is well known that nasal colonization with $S$. aureus along with MRSA, particularly in biofilm form, is associated with CRS disease recalcitrance and poor outcomes after sinus surgery [27-30]. Biofilms are thought to be the main mediators for disease persistence and treatment failure in various chronic disorders including CRS [30]. To our knowledge, no studies have investigated the anti-biofilm properties of commensal Corynebacteria, including C. accolens, against S. aureus and MRSA. However, Iwase and colleagues have previously shown the activity of another commensal nasal bacterium, S. epidermidis, in disrupting biofilm formation and previously established biofilms of $S$. aureus through the production of bioactive extracellular serine protease (Esp) [31]. In our study, CFCSs from selected C. accolens strains, C779, C781 and C787, showed a concentration-dependent inhibition of biofilms formed by $S$. aureus and MRSA CIs. Therefore, our findings support the potential use of $C$. accolens or bioactive compounds derived from those strains as antimicrobials against $S$. aureus biofilms.

\section{Materials and Methods}

\subsection{Collection of Clinical Isolates}

Ethics clearance for the collection, storage and use of clinical isolates was obtained from TQEH Human Research Ethics Committee (HREC/15/TQEH/132). All study subjects provided their written consent to participate in this study. Nasal swabs were collected at the time of surgery from 16 CRS patients (S. aureus clinical isolates) and from 20 non-CRS control patients (C. accolens clinical isolates) in a sterile Amies transport medium (Sigma Transwab, MWE Medical Wire, Corsham, UK), placed on ice and immediately transported to our research laboratory for processing.

S. aureus clinical isolates were identified from nasal swabs of CRS patients by culturing on mannitol salt agar (Oxoid, Basingstoke, UK) at $37^{\circ} \mathrm{C}$ overnight followed by specieslevel identification using API 20 Staph test system (bioMerieux, Australia) according to the manufacturer's instructions. All isolates were then screened for MRSA using a super sensitive and specific chromogenic MRSA selective agar $\left(\right.$ CHROMID $^{\circledR}$ MRSA SMART, bioMerieux, Australia) as described previously [32].

Non-CRS controls were patients undergoing septoplasty with no prior history of CRS, acute sinusitis, tonsillitis and ear infections in the 6 months prior to surgery. Nasal swabs in bacterial transport medium were first vortexed for $60 \mathrm{~s}$ and then diluted with phosphate buffer saline (PBS) 1:10. One-hundred-microliter aliquots of diluted samples were overlaid on Columbia agar plates with $5 \%$ sheep blood (Thermo Scientific, Oxoid, 
Australia) and incubated at $37^{\circ} \mathrm{C}$ for $48-72 \mathrm{~h}$. Cultures were inspected daily before colony identification, and visible bacterial colonies were subcultured onto tryptone soya agar (TSA) (Oxoid, Basingstoke, UK) supplemented with $0.8 \%$ Tween 80 and incubated for $48 \mathrm{~h}$ at $37^{\circ} \mathrm{C}$ with $5 \% \mathrm{CO}_{2}$ and screened phenotypically based on colony size and culture morphology. Biochemical characterization of the isolates was performed using the API Coryne test system (BioMérieux NSW, Australia) following the manufacturer's instructions. The isolates were stored at $-80{ }^{\circ} \mathrm{C}$ in tryptone soya broth (TSB) (Oxoid, Basingstoke, UK) plus 20\% $(v / v)$ sterile glycerol for further analysis.

\subsection{C. accolens Genomic DNA (gDNA) Extraction and DNA Quality Control}

Bacterial gDNA was extracted from a $48 \mathrm{hr}$ culture suspension of the isolates using a DNeasy Blood and Tissue kit (Qiagen, Hilden, Germany), following the manufacturer's recommendations. The concentration of DNA was determined by recording the absorbance at $260 \mathrm{~nm}\left(\mathrm{~A}_{260}\right)$ using a NanoDrop 2000 spectrophotometer (Thermo Scientific, Waltham, MA, USA). The DNA purity was determined from the optical density absorbance value; $\mathrm{A}_{260} / \mathrm{A}_{280} \mathrm{~nm}$ ratio. Moreover, the DNA integrity was evaluated through gel electrophoresis. Briefly, $5 \mu \mathrm{L}$ of each DNA extract was run on $1.8 \%$ agarose gel (Sigma-Aldrich, USA) in $1 \times$ Tris-Acetate-EDTA (TAE) buffer at 100 Volts for approximately 60 min and stained with $10,000 \times$ concentrate SYBR Safe (Invitrogen, Thermo Scientific, city, Canberra, Australia). DNA bands were visualized using the ChemiDoc ${ }^{\mathrm{TM}}$ Touch imaging system (Bio-Rad, NSW, Australia).

\subsection{Polymerase Chain Reaction (PCR) Amplification of Partial rpoB Gene}

PCR was carried out in a T100 TM Thermal cycler (Bio-Rad, NSW, Australia) using oligonucleotide primers, C2700F and C3130R (Table S1) according to a previously described protocol with little modification [18]. Briefly, amplification reactions were performed in a final volume of $50 \mu \mathrm{L}$ containing $5 \mu \mathrm{L}$ of $10 \times$ standard Taq Mg-free buffer, $6 \mu \mathrm{L}$ of $25 \mathrm{mM}$ $\mathrm{MgCl}_{2}$ solution, $1 \mu \mathrm{L}$ of $10 \mathrm{mM}$ dNTP mixture (dATP, dTTP, dGTP and dCTP), $0.25 \mu \mathrm{L}$ of $5.000 \mathrm{U} / \mathrm{mL}$ Taq DNA polymerase (all from BioLabs inc., Rowley, MA, USA), $1 \mu \mathrm{L}$ of $10 \mu \mathrm{M}$ concentration of each forward and reverse primer (Integrated DNA Technologies, SA, Australia), $25.75 \mu \mathrm{L}$ nuclease-free water and approximately $200 \mathrm{ng} / \mu \mathrm{L}$ of DNA adjusted to $10 \mu \mathrm{L}$ with nuclease-free water per reaction. Thereafter, PCR mixtures were subjected to 35 cycles of denaturation at $94{ }^{\circ} \mathrm{C}$ for $30 \mathrm{~s}$, primer annealing at $50.6{ }^{\circ} \mathrm{C}$ for $30 \mathrm{~s}$, and extension at $72{ }^{\circ} \mathrm{C}$ for $2 \mathrm{~min}$. A negative control (RNAse free water) and positive control (C. accolens ATCC49726, from American Type Culture Collection, Manassas, VA, USA) reaction were set up for every PCR experiment.

Amplified PCR products were separated on a 1.8\% agarose gel (Sigma-Aldrich, St. Louis, MO, USA) with $10 \mu \mathrm{L}$ of 10,000× concentrate SYBR Safe DNA gel stain (Invitrogen, Thermo Scientific, Canberra, Australia) in 1xTris-Acetate-EDTA (TAE) buffer at 100 Volts for $60 \mathrm{~min}$. The gels were visualized using ChemiDoc ${ }^{\mathrm{TM}}$ Touch imaging system (Bio-Rad). The size of PCR products was estimated by comparison with a $1 \mathrm{~kb}$ plus DNA ladder (BioLabs Ltd., Rowley, MA, USA). The primer sequences and amplicon size used for the detection of Corynebacteria are described in Supplemental Table S1.

\section{4. rpoB Gene Sequencing and Strain Identification of C. accolens}

The amplified PCR products were purified from agarose gel using QIAquick Gel extraction kits (Qiagen $\mathrm{GmbH}$, Hilden, Germany) following the manufacturer's extraction protocol. The concentration, purity and integrity of the recovered DNA samples were assessed using the NanoDrop 2000 spectrophotometer (Thermo Scientific, Waltham, MA, USA) and through agarose gel electrophoresis as specified. The purified DNA was then sent to the Australian Genome Research Facility Ltd. (AGRF) for sequencing. All samples were prepared for sequencing following the guide to AGRF sequencing service for Purified DNA (PD) as follows: 10 pmol of a primer (Forward or Reverse) + 12-18 ng of purified DNA + sterile MilliQ water (in a total volume of $12 \mu \mathrm{L}$ ). All sequencing results were 
analysed by comparing with NCBI GenBank database using the Blast program (http: / / blast.ncbi.nlm.nih.gov/Blast.cgi, accessed on 9 February 2021) for strain identification.

\subsection{Phylogenetic Analysis}

Based on the $r p o B$ gene sequence data, a phylogenetic tree elucidating the relationships between the identified strains was constructed. The nucleotide sequences were aligned through ClustalW program using MEGA (version 7.0) software, and evolutionary analysis was conducted using the neighbour-joining method keeping 1000 bootstrap replications [33]. The analysis involved 19 nucleotide sequences.

\subsection{Nucleotide Sequence Accession Numbers}

The rpoB sequences of $10 \mathrm{C}$. accolens strains, C778, C779, C780, C781, C782, C783, C784, C785, C786 and C787 have been deposited in the GenBank database (https:/ / www. ncbi.nlm.nih.gov / genbank, accessed on 30 December 2020), under the accession numbers MT856944, MT856945, MT856946, MT856947, MT856948, MT856949, MT856950, MT856951, MT856952 and MT856953, respectively.

\subsection{Deferred Growth Inhibition Assay}

The antagonistic activity of all $C$. accolens clinical strains and a culture collection strain, C. accolens ATCC 49726 was evaluated against S. aureus clinical isolates and S. aureus ATCC 25923 (from ATCC, Manassas, VA, USA) using deferred growth inhibition assays as described previously [34] with modifications. Briefly, a $48 \mathrm{~h} \mathrm{C}$. accolens culture ( $20 \mu \mathrm{L}$, approximately $10^{8}$ cells) in TSB (test inhibitor strains) was pipetted onto the centre of a TSA plate supplemented with $0.8 \%$ Tween 80 and incubated for $48 \mathrm{~h}$ at $37^{\circ} \mathrm{C}$ with $5 \% \mathrm{CO}_{2}$. Single colonies of a $24 \mathrm{~h}$ culture of $S$. aureus (competitor strains) were suspended in sterile $0.9 \%$ saline and standardized to McFarland units of 1.0 (approximately $3 \times 10^{8} \mathrm{CFU} / \mathrm{mL}$ ) followed by a dilution of 1:10 in TSB. Next, approximately $250 \mu \mathrm{L}$ of diluted culture were sprayed over the entire agar surface previously spotted with $C$. accolens and then incubated for a further 18-24 h. After incubation, a photograph was taken and the extent of the growth inhibition zone around the $C$. accolens spot was calculated quantitatively by measuring the diameter of the inhibition zone in millimetres minus the diameter of the central spot of the inhibitor strain. The test was done in triplicate, and the average of the diameters of the inhibition zones was obtained. The extent of inhibition was scored based on the inhibition zone diameter result as $-(0 \mathrm{~mm}),+(<5 \mathrm{~mm}),++(5-7 \mathrm{~mm}),+++(8-10 \mathrm{~mm})$ and $++++(>10 \mathrm{~mm})$.

\subsection{Preparation of Concentrated Cell-Free Culture Supernatants (CFCSs) from C. accolens Strains}

C. accolens strains were individually grown in $10 \mathrm{~mL}$ TSB in a shaking incubator at $37^{\circ} \mathrm{C}$ for $48 \mathrm{hrs}$. The CFCSs were obtained from $48 \mathrm{hr}$ cultures of C. accolens in TSB by centrifugation $\left(4000 \times g, 4{ }^{\circ} \mathrm{C}\right.$ for $\left.10 \mathrm{~min}\right)$ followed by filtration through $0.2 \mu \mathrm{m}$ sterile syringe filter (Pall Life Sciences, UK). Next, supernatants were passed through 3-kDa filter concentrator (Pierce Protein Concentrator, Thermo Fisher Scientific, Rockford, IL, USA) using centrifugation at $4000 \times g, 4{ }^{\circ} \mathrm{C}$ for $1-2 \mathrm{~h}$ to collect secreted proteins as described previously [35]. The protein concentration was then determined using Quick Start Bradford Protein Assay Kit (Bio-Rad Laboratories, USA) according to the manufacturer's protocol. All reactions were carried out in duplicate. Concentrated CFCS was then stored as singleuse aliquots at $-80{ }^{\circ} \mathrm{C}$ until use.

\subsection{Assessment of Anti-Bacterial Activity Using Concentrated CFCS and Minimum Inhibitory Treatment}

The inhibitory activity of CFCS from C. accolens isolates was tested against representative $S$. aureus and MRSA isolates following a broth micro-dilution assay protocol as described earlier [36] with minor modifications. The concentrated CFCS were first diluted in various concentration ranges $(30 \%, 50 \%, 70 \%$ and $90 \%)$ using TSB. Next, $198 \mu \mathrm{L}$ of 
the diluted mixture was dispensed in 96-well microtiter plates (Life Sciences, Boca Raton, FL, USA) to make CFCS with final concentrations of 102, 170, 238 and $306 \mu \mathrm{g} / \mathrm{mL}$. The inoculum was then prepared from all tested $S$. aureus isolates by suspending $18-24 \mathrm{~h}$ young colonies pre-cultured on TSA in $3 \mathrm{~mL}$ of sterile saline $(\mathrm{NaCl} 0.9 \% \mathrm{w} / \mathrm{v})$ and adjusted to $0.5 \mathrm{McFarland}$ turbidity standard $\left(1.0-2.0 \times 10^{8} \mathrm{CFU} / \mathrm{mL}\right)$. Following this, $2 \mu \mathrm{L}$ of bacterial suspension was inoculated to each well, and plates were incubated at $37^{\circ} \mathrm{C}$ for $24 \mathrm{~h}$. Wells containing bacteria without supernatant that was grown with the corresponding volume of TSB and sterile TSB-containing wells were used as positive and negative growth controls, respectively. After incubation, bacterial growth was determined by measuring the optical density (OD) at $595 \mathrm{~nm}$ using a microplate absorbance reader (iMark ${ }^{\mathrm{TM}}$, Bio-Rad, Australia). The inhibitory activity of the supernatant was calculated by comparing OD values between treated and untreated wells. The minimum inhibitory treatment was determined for 3 selected C. accolens strains' concentrated CFCSs (C. accolens C779, C. accolens C781 and C. accolens C787). The assays were performed in three replicates, and the antimicrobial activity results are expressed as mean ( \pm standard error of the means).

\subsection{Proteinase K and Heat Inactivation of CFCSs}

Inactivation experiments of CFCS were carried out using Proteinase $\mathrm{K}$ and heat treatment as previously described [37] with minor amendments. An aliquot of CFCS from selected C. accolens strains were treated with proteinase K (Sigma-Aldrich, St. Louis, MO, USA), at a final concentration of $1 \mathrm{mg} / \mathrm{mL}$ at $37^{\circ} \mathrm{C}$ for $5 \mathrm{~h}$. After incubation, the samples were subjected to heat treatment at $55^{\circ} \mathrm{C}$ for $30 \mathrm{~min}$ to inactivate protease enzymes. Next, the samples were allowed to cool to room temperature for $15 \mathrm{~min}$ before application. The antimicrobial activity of samples was then tested against representative $S$. aureus and MRSA clinical isolates and the reference strain S. aureus ATCC 25923 using a micro-dilution method in 96-well microtiter plates as specified. Proteinase K was used alone in the corresponding dilution broth (TSB) as a positive control, and wells containing TSB alone were used as a negative control. Three experimental replicates were performed for each protein sample, and data are presented as mean \pm SEM of the three experiments.

\subsection{Protein Clean-Up from CFCS and Detection of Anti-Bacterial Activity}

To remove salts and ionic contaminants such as detergents, lipids and phenolic compounds from CFCS, we used a 2-D Clean-Up Kit (GE-Biosciences, Piscataway, NJ, USA) according to the manufacturer's instructions. At completion of the washing steps, $50 \mu \mathrm{L}$ of sterile MilliQ water was added to resuspend the protein pellet. Following this, the anti-bacterial activity of purified protein samples (cleaned CFCS in sterile MilliQ water and TSB at a ratio of $30 \%, 50 \%, 70 \%$ and $90 \%(v / v))$ was tested against representative $S$. aureus and MRSA clinical isolates and S. aureus ATCC 25923 as specified. Controls were bacterial inoculum in TSB and sterile MilliQ water at identical volume ratios along with positive growth controls (bacterial inoculum in TSB) and a negative control (sterile TSB). Results are presented as the mean values \pm SEM of three independent experiments for each sample.

\subsection{Assessment of Anti-Biofilm Activity Using C. accolens CFCSs}

\subsubsection{Determination of Biofilm Metabolic Activity}

To assess the ability of $C$. accolens CFCS to inhibit the metabolic activity of $S$. aureus biofilms, alamarBlue biofilm assay was carried out using clear-bottom black 96-well plates as described previously [38]. Briefly, overnight cultures of $S$. aureus isolates grown in TSA were transferred into a sterile glass tube of $0.9 \%$ saline and adjusted to $1.0 \mathrm{McFarland}$ turbidity standard (approximately $3 \times 10^{8} \mathrm{CFU} / \mathrm{mL}$ ). Next, the suspension was diluted into TSB at 1:15 ratio, and $150 \mu \mathrm{L}$ of the final suspension was transferred to flat-bottom black 96-well microtiter plates and incubated at $37^{\circ} \mathrm{C}$ for $48 \mathrm{~h}$ in the dark on a rotating shaker to form biofilms. The wells were washed twice with $200 \mu \mathrm{L} 1 \times$ PBS to remove planktonic cells and air-dried for 5-10 min. Subsequently, wells were filled with $180 \mu \mathrm{L}$ of different concentrations of $C$. accolens CFCS diluted in TSB (30\%, 50\%, 70\% and 90\%) and incubated 
at $37^{\circ} \mathrm{C}$ on a rotating shaker for $24 \mathrm{~h}$ in the dark. Wells were then washed twice with $200 \mu \mathrm{L}$ $1 \times$ PBS and air-dried for 5-10 min. Next, plates were stained with $200 \mu \mathrm{L}$ alamarBlue (Invitrogen, Thermo Fisher Scientific, Hillsboro, OR, USA) and incubated for $3-5 \mathrm{~h}$ at $37^{\circ} \mathrm{C}$ on a rotating shaker. Wells containing bacterial culture without CFCS treatment and wells containing TSB without bacterial culture were included as a positive growth control and a sterility control, respectively. The fluorescence intensity of each well was then read every hour by a microplate reader FLUOstar OPTIMA (BMG LABTECH, Ortenberg, Germany) at a wavelength excitation $530 \mathrm{~nm}$ and emission $590 \mathrm{~nm}$ until maximum fluorescence was reached. Comparing the average fluorescence intensity (FI) of the growth control wells with that of the CFCS treated wells, the inhibition percentages (\% inhibition) of metabolic activity was calculated by the following formula: [(FI growth control - FI CFCS treated)/FI growth control] $\times 100$. This assay was performed in triplicate for each treatment.

\subsubsection{Determination of Biofilm Biomass}

Forty-eight-hour S. aureus biofilms treated with CFCS were washed twice with 1xPBS to remove the planktonic cells. The plates were then air-dried for $10 \mathrm{~min}$, and the surfaceattached biofilms were stained with $180 \mu \mathrm{L}$ of $0.1 \%(\mathrm{v} / \mathrm{v})$ crystal violet per well and incubated at room temperature for $15 \mathrm{~min}$. Subsequently, the crystal violet was removed, and the plates were washed three times with $200 \mu \mathrm{L}$ per well sterile MilliQ water to remove the unabsorbed stain. Next, $180 \mu \mathrm{L}$ per well $30 \%$ acetic acid was added and incubated on a plate shaker until the crystal violet solubilised. Stained biofilm biomass was determined by measuring absorbance at $595 \mathrm{~nm}$ using the microplate reader iMark $^{\mathrm{TM}}$, Bio-Rad, NSW, Australia). All experiments included a sterility control well containing TSB without bacterial culture and a growth control well (as 100\% cell mass) containing bacterial culture without CFCS treatment. The mean absorbance (Abs $595 \mathrm{~nm}$ ) of the samples was determined, and the percentage of biofilm biomass reduction by the CFCS was calculated by the following formula: [(Abs growth control - Abs CFCS treated)/Abs growth control] $\times 100$. All experiments were performed in triplicate and the mean value was calculated with the standard error.

\subsection{Statistical Analysis}

All the measurements were performed in triplicate, and the values were expressed as mean \pm standard error of the mean (SEM). The mean differences in absorbance value between CFCS treated and growth control wells were compared and analysed by One-way analysis of variance (ANOVA) using Dunnett's multiple comparisons test for anti-bacterial and anti-biofilm assays. All experimental data analyses were performed in GraphPad Prism software version 8.0 (GraphPad Software, San Diego, CA, USA). Statistical significance was determined at $p$-value $<0.05$.

\section{Conclusions}

Taken together, the antimicrobial activity of C. accolens strains and their secreted proteins against $S$. aureus and MRSA clinical isolates in planktonic and biofilm form could be useful in the prevention of $S$. aureus outgrowth in the nasal microbiota and opens the possibility for a protective use of Corynebacteria against antibiotic-resistant $S$. aureus nasal colonization in a complex niche. Our findings have potential clinical implications towards the development of personalized probiotic therapy and might contribute to shaping the disrupted nasal microbiota in CRS.

Supplementary Materials: The following are available online at https:/ / www.mdpi.com/2076-081 7/10/2/207/s1, Table S1: Description of oligonucleotides used for PCR amplification of rpoB gene in C. accolens isolates, Table S2: Pathogenic S. aureus strains (8MSSA and 8MRSA) isolated from the sinonasal cavity of CRS patients used in this study, Figure S1: Identification of Corynebacterium accolens isolates by PCR amplification of partial rpoB gene (446-bp fragment). 
Author Contributions: Project conceptualization, M.A.M., A.J.H. and S.V.; experimental design and laboratory investigation, M.A.M., S.L. and C.C.; data management and statistical analysis, M.A.M. and S.L.; project administration and supervision, S.V., P.-J.W. and A.J.P.; funding acquisition, A.J.P. and S.V.; manuscript draft preparation and revision, M.A.M.; manuscript review and editing, A.J.H., S.L., C.C., A.J.P., P.-J.W. and S.V. All authors have read and agreed to the published version of the manuscript.

Funding: This research was supported from Garnett Passe and Rodney Williams Memorial Foundation Conjoint Grant awarded to A.J.P. and S.V; and the University of Adelaide (International Scholarship) to M.A.M.

Institutional Review Board Statement: The study was conducted according to the guidelines of the Declaration of Helsinki, and approved by the Human Research Ethics Committee of TQEH (HREC/15/TQEH/132), approved on 18 August 2015.

Informed Consent Statement: Informed consent was obtained from all subjects involved in the study.

Data Availability Statement: The data presented in this study are available on request from the corresponding author.

Acknowledgments: We are grateful to Mahnaz Ramezanpour (Department of Otolaryngology-Head and Neck Surgery, Basil Hetzel Institute for Translational Health Research, University of Adelaide, Australia) for her technical support and constructive guidance.

Conflicts of Interest: The authors declare no conflict of interest.

\section{References}

1. Riechelmann, H. Europäischen Akademie für Allergie und Klinische Immunologie (EAACI) un der European Rhinologic Society (ERS)—Chronic Rhinosinusitis. EPOS 2012 Part I]. Laryngorhinootologie 2013, 92, 193-201. [PubMed]

2. Hoggard, M.; Biswas, K.; Zoing, M.; Mackenzie, B.W.; Taylor, M.W.; Douglas, R.G. Evidence of microbiota dysbiosis in chronic rhinosinusitis. Int. Forum Allergy Rhinol. 2017, 7, 230-239. [CrossRef] [PubMed]

3. Copeland, E.; Leonard, K.; Carney, R.; Kong, J.; Forer, M.; Naidoo, Y.; Oliver, B.G.G.; Seymour, J.R.; Woodcock, S.; Burke, C.M.; et al. Chronic rhinosinusitis: Potential role of microbial dysbiosis and recommendations for sampling sites. Front. Cell. Infect. Microbiol. 2018, 8, 57. [CrossRef] [PubMed]

4. Petersen, C.; Round, J.L. Defining dysbiosis and its influence on host immunity and disease. Cell. Microbiol. 2014, 16, 1024-1033. [CrossRef] [PubMed]

5. Paramasivan, S.; Bassiouni, A.; Shiffer, A.; Dillon, M.R.; Cope, E.K.; Cooksley, C.; Ramezanpour, M.; Moraitis, S.; Ali, M.J.; Bleier B.; et al. The international sinonasal microbiome study: A multicentre, multinational characterization of sinonasal bacterial ecology. Allergy 2020, 75, 2037-2049. [CrossRef]

6. Cleland, E.J.; Bassiouni, A.; Wormald, P.J. The bacteriology of chronic rhinosinusitis and the pre-eminence of Staphylococcus aureus in revision patients. Int. Forum Allergy Rhinol. 2013, 3, 862-866. [CrossRef]

7. Vickery, T.W.; Ramakrishnan, V.R.; Suh, J.D. The role of Staphylococcus aureus in patients with chronic sinusitis and nasal polyposis. Curr. Allergy Asthma Rep. 2019, 19, 21. [CrossRef]

8. Fokkens, W.J.; Lund, V.J.; Mullol, J.; Bachert, C.; Alobid, I.; Baroody, F.; Cohen, N.; Cervin, A.; Douglas, R.; Gevaert, P.; et al. European position paper on rhinosinusitis and nasal polyps 2012. Rhinology 2012, 23, 1-12.

9. Foreman, A.; Wormald, P.J. Different biofilms, different disease? A clinical outcomes study. Laryngoscope 2010, 120, 1701-1706. [CrossRef]

10. Stubbendieck, R.M.; Straight, P.D. Multifaceted interfaces of bacterial competition. J. Bacteriol. 2016, 198, 2145-2155. [CrossRef]

11. Hibbing, M.E.; Fuqua, C.; Parsek, M.R.; Peterson, S.B. Bacterial competition: Surviving and thriving in the microbial jungle. Nat. Rev. Microbiol. 2010, 8, 15. [CrossRef]

12. Speert, D.P.; Wannamaker, L.W.; Gray, E.D.; Clawson, C.C. Bactericidal effect of oleic acid on group A streptococci: Mechanism of action. Infect. Immun. 1979, 26, 1202-1210. [CrossRef] [PubMed]

13. Bomar, L.; Brugger, S.D.; Yost, B.H.; Davies, S.S.; Lemon, K.P. Corynebacterium accolens releases antipneumococcal free fatty acids from human nostril and skin surface triacylglycerols. MBio 2016, 7, e01725-15. [CrossRef] [PubMed]

14. Kechagia, M.; Basoulis, D.; Konstantopoulou, S.; Dimitriadi, D.; Gyftopoulou, K.; Skarmoutsou, N.; Fakiri, E.M. Health benefits of probiotics: A review. ISRN Nutr. 2013, 2013, 481651. [CrossRef]

15. Cleland, E.J.; Drilling, A.; Bassiouni, A.; James, C.; Vreugde, S.; Wormald, P.-J. Probiotic manipulation of the chronic rhinosinusitis microbiome. Int. Forum Allergy Rhinol. 2014, 309-314. [CrossRef]

16. Abreu, N.A.; Nagalingam, N.A.; Song, Y.; Roediger, F.C.; Pletcher, S.D.; Goldberg, A.N.; Lynch, S.V. Sinus microbiome diversity depletion and Corynebacterium tuberculostearicum enrichment mediates rhinosinusitis. Sci. Transl. Med. 2012, 4, 151ra124. [CrossRef] [PubMed] 
17. Uehara, Y.; Nakama, H.; Agematsu, K.; Uchida, M.; Kawakami, Y.; Fattah, A.A.; Maruchi, N. Bacterial interference among nasal inhabitants: Eradication of Staphylococcus aureus from nasal cavities by artificial implantation of Corynebacterium sp. J. Hosp. Infect. 2000, 44, 127-133. [CrossRef]

18. Khamis, A.D.; Raoult, D.; La Scola, B. rpoB gene sequencing for identification of Corynebacterium species. J. Clin. Microbiol. 2004, 42, 3925-3931. [CrossRef]

19. Desrosiers, M.; Valera, F.C.P. Brave New (Microbial) World: Implications for nasal and sinus disorders. Braz. J. Otorhinolaryngol. 2019, 85, 675-677. [CrossRef]

20. De Boeck, I.; Wittouck, S.; Martens, K.; Claes, J.; Jorissen, M.; Steelant, B.; van den Broek, M.F.L.; Seys, S.F.; Hellings, P.W.; Vanderveken, O.M.; et al. Anterior nares diversity and pathobionts represent sinus microbiome in chronic rhinosinusitis. MSphere 2019, 4. [CrossRef]

21. Psaltis, A.J.; Wormald, P.-J. Therapy of sinonasal microbiome in CRS: A critical approach. Curr. Allergy Asthma Rep. 2017, 17, 59. [CrossRef]

22. Khamis, A.D.; Raoult, D.; La Scola, B. Comparison between rpoB and $16 \mathrm{~S}$ rRNA gene sequencing for molecular identification of 168 clinical isolates of Corynebacterium. J. Clin. Microbiol. 2005, 43, 1934-1936. [CrossRef]

23. Wos-Oxley, M.L.; Plumeier, I.; Von Eiff, C.; Taudien, S.; Platzer, M.; Vilchez-Vargas, R.; Becker, K.; Pieper, D.H. A poke into the diversity and associations within human anterior nare microbial communities. ISME J. 2010, 4, 839-851. [CrossRef]

24. Hardy, B.L.; Dickey, S.W.; Plaut, R.D.; Riggins, D.P.; Stibitz, S.; Otto, M.; Merrell, D.S. Corynebacterium pseudodiphtheriticum exploits Staphylococcus aureus virulence components in a novel polymicrobial defense strategy. MBio 2019, 10, e02491-18. [CrossRef]

25. Ramsey, M.M.; Freire, M.O.; Gabrilska, R.A.; Rumbaugh, K.P.; Lemon, K.P. Staphylococcus aureus shifts toward commensalism in response to Corynebacterium species. Front. Microbiol. 2016, 7, 1230. [CrossRef]

26. Bitschar, K.; Sauer, B.; Focken, J.; Dehmer, H.; Moos, S.; Konnerth, M.; Schilling, N.A.; Grond, S.; Kalbacher, H.; Kurschus, F.C.; et al. Lugdunin amplifies innate immune responses in the skin in synergy with host-and microbiota-derived factors. Nat. Commun. 2019, 10, 1-14. [CrossRef]

27. Thirumazhisi Sachithanandam, S. Rising methicillin-resistant Staphylococcus aureus infections in ear, nose, and throat diseases. Case Rep. Otolaryngol. 2014, 2014. [CrossRef]

28. Psaltis, A.J.; Weitzel, E.K.; Ha, K.R.; Wormald, P.-J. The effect of bacterial biofilms on post-sinus surgical outcomes. Am. J. Rhinol. 2008, 22, 1-6. [CrossRef] [PubMed]

29. Singh, P.; Mehta, R.; Agarwal, S.; Mishra, P. Bacterial biofilm on the sinus mucosa of healthy subjects and patients with chronic rhinosinusitis (with or without nasal polyposis). J. Laryngol. Otol. 2015, 129, 46-49. [CrossRef] [PubMed]

30. Richter, K. Tackling superbugs in their slime castles: Innovative approaches against antimicrobial-resistant biofilm infections. Microbiol. Aust. 2019, 40, 165-168. [CrossRef]

31. Iwase, T.; Uehara, Y.; Shinji, H.; Tajima, A.; Seo, H.; Takada, K.; Agata, T.; Mizunoe, Y. Staphylococcus epidermidis Esp inhibits Staphylococcus aureus biofilm formation and nasal colonization. Nature 2010, 465, 346-349. [CrossRef]

32. Dodémont, M.; Verhulst, C.; Nonhoff, C.; Nagant, C.; Denis, O.; Kluytmans, J. Prospective two-center comparison of three chromogenic agars for methicillin-resistant Staphylococcus aureus screening in hospitalized patients. J. Clin. Microbiol. 2015, 53, 3014-3016. [CrossRef]

33. Saitou, N.; Nei, M. The neighbor-joining method: A new method for reconstructing phylogenetic trees. Mol. Biol. Evol. 1987, 4, 406-425. [PubMed]

34. Moran, J.C.; Crank, E.L.; Ghabban, H.A.; Horsburgh, M.J. Deferred growth inhibition assay to quantify the effect of bacteriaderived antimicrobials on competition. J. Vis. Exp. 2016, e54437. [CrossRef]

35. Panchatcharam, B.S.; Cooksley, C.M.; Ramezanpour, M.; Vediappan, R.S.; Bassiouni, A.; Wormald, P.J.; Psaltis, A.J.; Vreugde, S. Staphylococcus aureus biofilm exoproteins are cytotoxic to human nasal epithelial barrier in chronic rhinosinusitis. Int. Forum Allergy Rhinol. 2020. [CrossRef] [PubMed]

36. Inturri, R.; Trovato, L.; Volti, G.L.; Oliveri, S.; Blandino, G.; Trovato, L. In vitro inhibitory activity of Bifidobacterium longum BB536 and Lactobacillus rhamnosus HN001 alone or in combination against bacterial and Candida reference strains and clinical isolates. Heliyon 2019, 5, e02891. [CrossRef] [PubMed]

37. Al Akeel, R.; Mateen, A.; Alharbi, K.K.; Alyousef, A.A.; Al-Mandeel, H.M.; Syed, R. Purification and MIC analysis of antimicrobial proteins from Cucumis sativus L. seeds. BMC Complement. Altern. Med. 2018, 18, 1-6. [CrossRef]

38. Richter, K.; Ramezanpour, M.; Thomas, N.; Prestidge, C.A.; Wprmald, P.-J.; Vreugde, S. Mind "De GaPP": In vitro efficacy of deferiprone and gallium-protoporphyrin against Staphylococcus aureus biofilms. Int. Forum Allergy Rhinol. 2016, 6, 737-743. [CrossRef] [PubMed] 